\title{
Measurement of Bed Turning and Comparison with Age, Gender, and Body Mass Index in a Healthy Population: Application of a Novel Mobility Detection System
}

\author{
Shang-Lin Chiang, ${ }^{1,2,3}$ Chia-Huei Lin, ${ }^{4,5}$ Shin-Tsu Chang, ${ }^{2,6}$ Chueh-Ho Lin, ${ }^{1}$ \\ Po-Yin Chen, ${ }^{1}$ Wen-Hsu Sung, ${ }^{1}$ and Shun-Hwa Wei ${ }^{1}$ \\ ${ }^{1}$ Department of Physical Therapy and Assistive Technology, National Yang Ming University, No. 155, Sec. 2, Linong Street, \\ Beitou, Taipei City 112, Taiwan \\ ${ }^{2}$ Department of Physical Medicine and Rehabilitation, School of Medicine, National Defense Medical Center, No. 325, Sec. 2, \\ Chenggong Road, Neihu District, Taipei City 114, Taiwan \\ ${ }^{3}$ Department of Physical Medicine and Rehabilitation, Tri-Service General Hospital, No. 325, Sec. 2, Chenggong Road, \\ Neihu District, Taipei City 114, Taiwan \\ ${ }^{4}$ Graduate Institute of Medical Sciences, National Defense Medical Center, No. 325, Sec. 2, Chenggong Road, \\ Neihu District, Taipei City 114, Taiwan \\ ${ }^{5}$ Department of Nursing, Tri-Service General Hospital, School of Nursing, National Defense Medical Center, No. 325, Sec. 2, \\ Chenggong Road, Neihu District, Taipei City 114, Taiwan \\ ${ }^{6}$ Department of Physical Medicine and Rehabilitation, Taichung Veterans General Hospital, No. 1650, Sec. 4, Taiwan Boulevard, \\ Xitun District, Taichung City 407, Taiwan
}

Correspondence should be addressed to Wen-Hsu Sung; wenhsusung@gmail.com and Shun-Hwa Wei; shunhwaw@gmail.com

Received 18 December 2013; Accepted 28 March 2014; Published 29 April 2014

Academic Editor: Ali I. Abdalla

Copyright (C) 2014 Shang-Lin Chiang et al. This is an open access article distributed under the Creative Commons Attribution License, which permits unrestricted use, distribution, and reproduction in any medium, provided the original work is properly cited.

We developed a mobility detection system to analyze pressure changes over time during side-turns in 29 healthy volunteers (17 males and 12 females) with a mean age of $46.1 \pm 19.64$ years (ranging from 23 to 86 years) in order to determine the effect of gender, age, and BMI on performance during bed postural change. Center of gravity (COG) location, peak pressure of counteraction, and time to reach peak pressure were the main outcomes used to gauge the ability to make a spontaneous side-turn. Men exhibited significantly higher side-turning force $(P=0.002)$ and back-turning force $(P=0.002)$ compared with women. Subjects with BMI $\geq 27 \mathrm{~kg} / \mathrm{m}^{2}$ had significantly higher side-turning force $(P=0.007)$ and back-turning force $(P=0.007)$ compared with those with $\mathrm{BMI}<27 \mathrm{~kg} / \mathrm{m}^{2}$. After adjusting for other covariates, age positively correlated with back-turning time $(P=0.033)$ and negatively correlated with side-turning speed $(P=0.005)$, back-turning speed $(P=0.014)$, side-turning force $(P=0.010)$, and back-turning force $(P=0.016)$, respectively. Turning times negatively correlated with time to reach peak pressure $(P=0.008)$. Our system was effective in detecting changes in turning swiftness in the bed-ridden subject.

\section{Introduction}

Determination of body position on a mattress is important for both management and prevention of bedsores in the bedridden patient and prevention of muscle atrophy in stroke patients. The development of pressure ulcers is a significant health concern, especially in the elderly population, as pressure ulcers can lead to serious systemic infections $[1,2]$.
Approximately 60,000 people each year die from complications related to pressure ulcers $[3,4]$.

Periodic postural change is the key to prevention of deep tissue injury and subsequent pressure ulcer development. However, to our knowledge, there is no objective technology available to evaluate turning ability. In addition, postural training and monitoring of turning motion recovery are needed in the elderly, the stroke patient, and the disabled. 


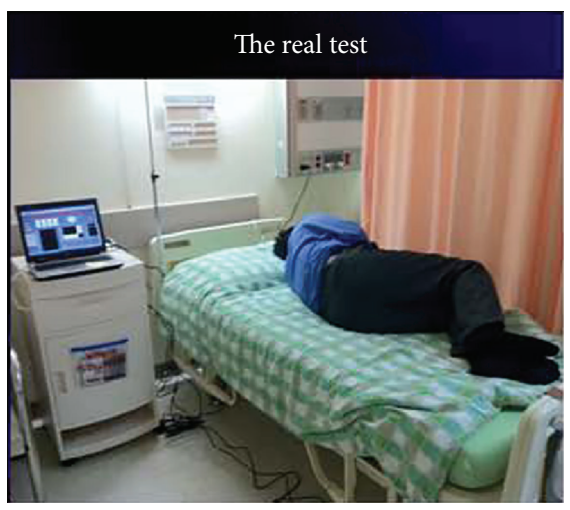

(a)

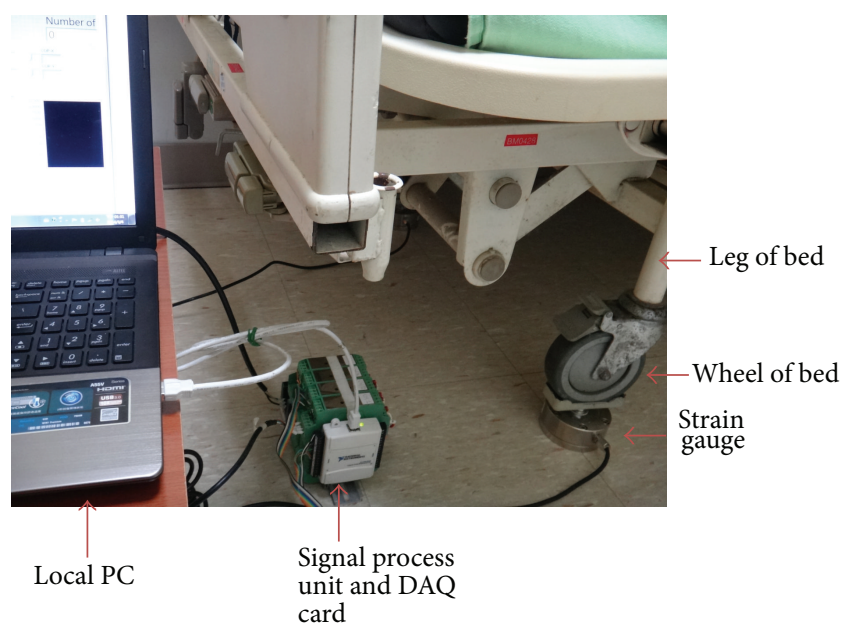

(b)

Figure 1: (a) The actual testing apparatus. (b) Close-up showing the sensor attached to the bed leg.

At present, patient body position is measured by visual inspection or by analysis of either a video monitoring system or expensive, high-resolution arrays of pressure sensors [57]. Due to high cost and time constraints on nursing care, often a set of accelerometers tethered to the patient is used as an alternative [3, 8-13]. Brown et al. measured postural changes, including lying, sitting, standing, or walking, by attaching wireless accelerometers to the ipsilateral thigh and ankle [8]. These accelerometers monitor a patient's position based on movement and position of the limbs and could potentially measure different levels of mobility that occur during hospitalization. Bagalà et al. proposed a single bodyfixed inertial sensor to provide quantitative description of the lie-to-sit-to-stand-to-walk [13]. Wireless accelerometers have also been used to measure turning in bed when attached to the thigh [9]. A wearable alternative, the so-called "Wearable Wireless Identification and Sensing Platform" or WISP, was designed with a single kinematic sensor capable of realtime monitoring to automatically detect bed entry and exit events. Located over the sternum, the WISP method is the preferred method to detect bed entry and exit in order to avoid falls which commonly occur near the bed in hospitals and residential care facilities [12]. To overcome the challenges faced by accelerometer-based monitoring, that is, a low accuracy, inability to recognize both posture and transportation mode simultaneously, and high computational complexity, Zhang and Poslad proposed a new GPS and foot-force sensor method which combines wearable FF sensors with GPS monitoring via a mobile phone [11]. Overall, accelerometry-based wearable motion detectors have been used for classification of posture and movement, estimation of energy expenditure, fall detection, and balance control evaluation [10] but cannot provide information regarding turning ability in bed.

We designed a low-cost mobility detection system not only to monitor spontaneous bed turning but also to assess turning ability in the bed-ridden patient. This mobility detection system combined a sensor mat (using a minimum number of sensors) with data analysis software to monitor turning ability. This system analyzed pressure changes over time during side-turns in 29 healthy subjects. Gravity center location and velocity during movement, time to complete a side-turn, peak counteraction pressure, and time to reach peak pressure (turning swiftness) were the parameters used to gauge the ability to make a spontaneous side-turn in bed.

\section{Subjects and Methods}

2.1. Subjects. This study was approved by the Institutional Review Board of Tri-Service General Hospital, National Defense Medical Center. All subjects gave their written informed consent prior to participation in the study.

In this study, normal healthy volunteers were asked to lie in the center of the bed and listen to instructions regarding bed turning (Figures 1(a) and 1(b)). Each subject was asked to turn first to their left side, then turn up, wait $10 \mathrm{sec}$, and then return to lying on their back and wait an additional $10 \mathrm{sec}$. This task was considered one complete turn and repeated 10 times. After the above task was completed, the same volunteer was asked to turn to the right and then turn up, and this task was repeated 10 times. Thus, for every test, the volunteer performed 20 turns. In order for the subject to recognize when to start and stop each motion, the physician gave clear orders for each turn, such as "turn left," "turn up," and so forth.

Data was collected over 20 turns back and forth for each subject. The force generated on the bed surface was recorded and analyzed, as described below.

2.2. Mobility Detection System. The mobility detection system was composed of four strain gauges (LFS1CC $150 \mathrm{~kg}$, Delta Transducers Co.) and additional data analysis software (Figure 2). The strain gauges were installed at each of the four legs of a bed (Figure 1(b)). When the subject rolled up onto his or her side, the change in center of pressure was recorded 


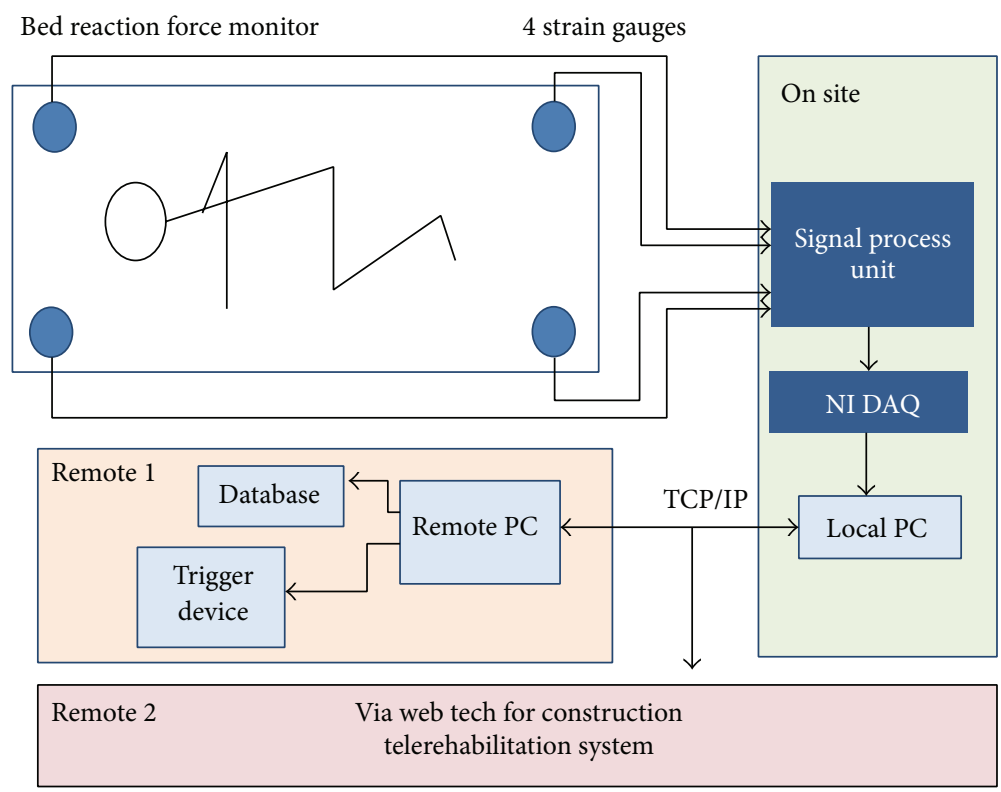

FIgURE 2: The mobility detection system (patent number M43319-Taiwan).

by each of the four strain gauges. We used lever principle to calculate the transposition of force in $X Y$ plane, as follows:

$$
\begin{gathered}
\sum F z=W=W 1+W 2+W 3+W 4, \\
\sum M y: x W=x 1 W 1+x 2 W 2+x 3 W 3+x 4 W 4, \\
\sum M x: y W=y 1 W 1+y 2 W 2+y 3 W 3+y 4 W 4, \\
\operatorname{coF} x \cong \frac{M y}{F z} ; \quad \operatorname{coF} y \cong \frac{M x}{F z},
\end{gathered}
$$

As graphically depicted in Figure 3, the sum of the changes in pressure readings from the four strain gauges was used to calculate the change in location of the subject on the bed over time (change in the center of pressure or center of force $(\mathrm{coF})$ ) using the following equation:

$$
\Delta \mathrm{coF}=\Delta(c x, y),
$$

where

$$
\begin{gathered}
c x(\text { center of horizontal force })=\frac{\sum a i x * F i}{\sum F i} \quad(i=1 \text { to } 4), \\
c y(\text { center of vertical force })=\frac{\sum a i y * F i}{\sum F i} \quad(i=1 \text { to } 4) .
\end{gathered}
$$

$\sum a i x * F i$ refers to the four load cells to withstand the forces generated on the $X$-axis to the total moment, and aix is the $x$-coordinate of four load cells, $\sum a i y * F i$ refers to the four load cells to withstand the forces generated on the $Y$-axis to the total moment, and aiy is the $y$-coordinate of four load cells.

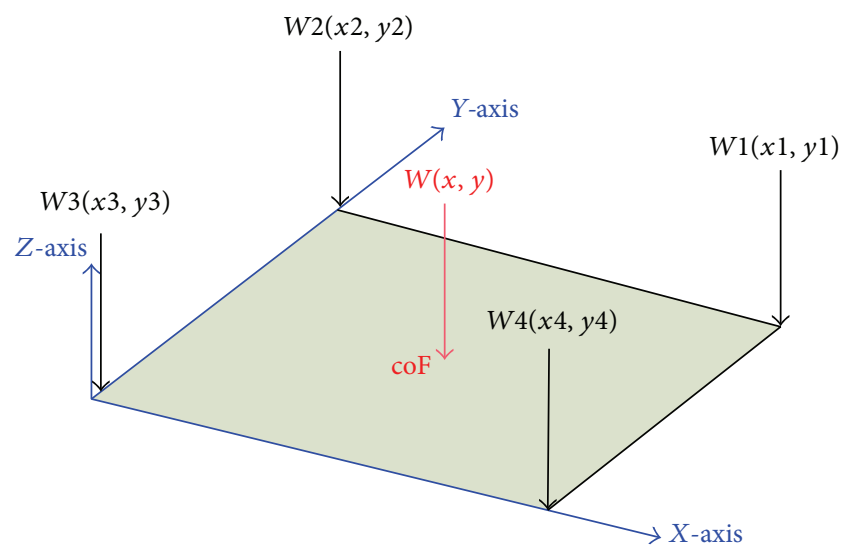

FIGURE 3: The sum of the changes in pressure readings from the four strain gauges is used to calculate the change in location of the subject on the bed over time (change in the center of pressure or center of force ( $\mathrm{coF})$ ).

The analog pressure readings were sent to a signal processing unit and then to the NI-DAQmx8.9 (National Instruments Corp., Austin, TX, USA) which performed analog to digital conversion. The resulting digital signals were input to a PC for data analysis (Figure 2). The data analysis program was written in Labview 2010 (National Instruments Corporation, Austin, TX, USA). The maximum sampling frequency was $100 \mathrm{~Hz}$, and the maximum voltage range was $\pm 10 \mathrm{~V}$. The choice of $100 \mathrm{~Hz}$ for the frequency was based on a mean turn time of $5.7 \mathrm{sec}$. It was felt that $100 \mathrm{~Hz}$ was sufficient frequency to monitor the turn time. In addition, if we used $1000 \mathrm{~Hz}$, it would have resulted in the accumulation of an enormous amount of data while monitoring over extended periods such as 24 hour observations. This system was also designed to 
allow for additional monitoring of multiple beds and remote monitoring, if needed, using the internet.

In summary, this study was designed to monitor coF or center of gravity (COG) changes. To clarify, (i) velocity of COG is equivalent to "turn/back speed," (ii) peak pressure of counteraction is equivalent to "turn/back force," and (iii) time to reach peak pressure is equivalent to "turn/back peak time." Main outcomes of the study were velocity of COG, peak pressure of counteraction, and time to reach peak pressure (turn/ back speed, turn/back force, and turn/back peak time). Secondary outcomes included movement distance of COG, sideturn time, and location of COG (TL/BL, turn time/back time, turn/back-weight ratio).

2.3. Parameter Definitions. The following terms were used throughout the study.

Turn time (sec) or side-turn time refers to the time to complete the left (or right) side-turn from the back position (resting position); TL $(\mathrm{cm})$ or movement distance of COG refers to gravity distance traveled to complete the left (or right) side-turn; turn speed $(\mathrm{cm} / \mathrm{sec})$ or velocity of COG refers to the speed used to complete the left (or right) sideturn and was equal to TL divided by turn time; turn peak time (ds) or time to reach peak pressure refers to turning swiftness when completing a side-turn; turn force $\left(\mathrm{kg} \cdot \mathrm{m} / \mathrm{s}^{2}\right)$ or peak pressure of counteraction refers to maximum reaction force expended to complete either the left (or right) side-turn; back time (sec) or side-turn time refers to time to return to one's back from either the left (or right) side-turn; $\mathrm{BL}(\mathrm{cm})$ or movement distance of COG refers to distance traveled upon returning to one's back after completing the left (or right) side-turn; back speed $(\mathrm{cm} / \mathrm{sec})$ or velocity of COG refers to the speed of turning onto one's back from either the left (or right) side-turn; back peak time (ds) or time to reach peak pressure refers to turning swiftness when completing a backturn; back force $\left(\mathrm{kg} \cdot \mathrm{m} / \mathrm{s}^{2}\right)$ or peak pressure of counteraction refers to maximum reaction force expended to complete the turn onto one's back from either the left (or right) side-turn; turn-weight ratio or location of COG refers to the ratio of turn force to weight; back-weight ratio or location of COG refers to the ratio of back force to weight.

2.4. Force Sensor Reliability and Validity. Using a $30 \mathrm{~kg}$ standard, each point of the test was repeated ten times, and the test-retest reliability ( 0.947 for position detected and 0.986 for weight measured) and concurrent validity ( 0.927 for position detected and 0.924 for weight measured) of the results were tested with regard to location and the weight measured, respectively. Both validity and reliability were found to be above 0.9. According to a systematic review by Wind et al. [14], the criteria of a high test-retest reliability and a high concurrent validity were above $0.8(r>0.8)$ and $0.75(r>0.75)$, respectively. The force sensor used in this study met both criteria with high validity and reliability.

2.5. Statistical Analysis. Continuous variables were summarized using mean \pm SD and categorical variables were expressed as frequencies and percentages. The intrarater
TABLE 1: Subject demographics.

\begin{tabular}{lc}
\hline Variables & $N=29$ \\
\hline Age & $46.1 \pm 19.64$ \\
Mean \pm SD & $23-86$ \\
Range & \\
Gender, $n(\%)$ & $17(58.6)$ \\
Male & $12(41.4)$ \\
Female & \\
Height $(\mathrm{cm})$ & $163.5 \pm 6.9$ \\
Mean \pm SD & $150-181$ \\
Range & \\
Weight $(\mathrm{cm})$ & $63.66 \pm 12.13$ \\
Mean \pm SD & $45-88$ \\
Range & \\
BMI $\left(\mathrm{kg} / \mathrm{m}^{2}\right)$ & $23.68 \pm 3.49$ \\
Mean \pm SD & $17.15-31.55$ \\
Range &
\end{tabular}

BMI: body mass index.

reliability of parameters that were used to evaluate turning ability was measured by intraclass correlation coefficients (ICCs). The repeated measures analysis of variance (ANOVA) was used to detect the within-subject (side-turning) and between-subject effects (gender, age, and BMI) on various parameters related to turning ability. To investigate the factors associated with turning ability, the generalized estimating equation (GEE) model, with an identity link function, was applied to accommodate the correlated data of repeat measurements within the same subjects. All statistical analyses were performed using SAS software version 9.2 (SAS Institute Inc., Cary, NC). A two-tailed $P<0.05$ indicated statistical significance.

\section{Results}

3.1. Subject Demographics. A total of 29 healthy volunteers, consisting of 17 males (58.6\%) and 12 females (41.4\%), were enrolled in the study. Their demographics are summarized in Table 1 . The mean age of the subjects was $46.1 \pm 19.64$ years (ranging from 23 to 86 years). Their average body height and body weight were $163.5 \pm 6.9 \mathrm{~cm}$ and $63.66 \pm 12.13 \mathrm{~kg}$, respectively. The average BMI was $23.68 \pm 3.49 \mathrm{~kg} / \mathrm{m}^{2}$ (ranging from 17.15 to $31.55 \mathrm{~kg} / \mathrm{m}^{2}$ ).

3.2. Intrarater Reliability. For evaluation of intrarater reliability, a separate pilot study of 10 subjects (not involving the 29 subjects used in this study) was conducted and 20 repeat measurements per each parameter (turn time, TL, turn speed, turn peak time, turn force, back time, BL, back speed, back peak time, back force, turn-weight ratio, and back-weight ratio) were taken by one physician (rater) for each subject.

Before the main study began, we wished to investigate reliability of these parameters for measuring turning ability within the same physician (rater). The intrarater reliability of parameters that describe turning ability was quantified using 
TABLE 2: Turning ability parameters.

\begin{tabular}{|c|c|c|c|c|c|}
\hline & \multicolumn{2}{|c|}{ Left side-turn } & \multicolumn{2}{|c|}{ Right side-turn } & \multirow[b]{2}{*}{$P$-value ${ }^{\dagger}$} \\
\hline & $n^{\mathrm{a}}$ & Mean \pm SD & $n$ & Mean \pm SD & \\
\hline TURN Time (sec) & 290 & $5.71 \pm 0.75$ & 290 & $5.75 \pm 1.27$ & 0.797 \\
\hline $\mathrm{TL}(\mathrm{cm})$ & 290 & $24.35 \pm 8.48$ & 290 & $24.46 \pm 8.68$ & 0.871 \\
\hline TURN Speed $(\mathrm{cm} / \mathrm{sec})$ & 290 & $4.31 \pm 1.57$ & 290 & $4.37 \pm 1.72$ & 0.551 \\
\hline TURN Peak Time (ds) & 290 & $8.44 \pm 7.65$ & 290 & $8.06 \pm 6.99$ & 0.750 \\
\hline TURN Force $\left(\mathrm{kg} \cdot \mathrm{m} / \mathrm{s}^{2}\right)$ & 290 & $64.49 \pm 13.10$ & 290 & $65.14 \pm 12.87$ & 0.448 \\
\hline BACK Time (sec) & 290 & $5.17 \pm 1.39$ & 290 & $5.29 \pm 1.87$ & 0.533 \\
\hline $\mathrm{BL}(\mathrm{cm})$ & 290 & $24.66 \pm 8.46$ & 290 & $24.64 \pm 8.77$ & 0.974 \\
\hline BACK Speed $(\mathrm{cm} / \mathrm{sec})$ & 290 & $4.89 \pm 1.72$ & 290 & $4.84 \pm 1.94$ & 0.754 \\
\hline BACK Peak Time (ds) & 290 & $11.14 \pm 13.76$ & 290 & $10.90 \pm 6.38$ & 0.862 \\
\hline BACK Force $\left(\mathrm{kg} \cdot \mathrm{m} / \mathrm{s}^{2}\right)$ & 290 & $65.03 \pm 13.50$ & 290 & $65.87 \pm 13.25$ & 0.183 \\
\hline TURN Weight ratio & 290 & $1.015 \pm 0.103$ & 290 & $1.031 \pm 0.139$ & 0.320 \\
\hline BACK Weight ratio & 290 & $1.024 \pm 0.115$ & 290 & $1.041 \pm 0.132$ & 0.129 \\
\hline
\end{tabular}

${ }^{a}$ Numbers of measurements; ${ }^{\dagger}$ determined by repeated measures ANOVA (within-subject effect).

TURN Time (sec): time to complete left (or right) side-turn from the back position (resting position).

TL $(\mathrm{cm})$ : distance traveled to complete left (or right) side-turn.

TURN Speed ( $\mathrm{cm} / \mathrm{sec}$ ): speed (distance/time) used to complete left (or right) side-turn.

TURN Peak Time (ds): time to reach peak pressure (turning swiftness) when completing side-turn.

TURN Force $\left(\mathrm{kg} \cdot \mathrm{m} / \mathrm{s}^{2}\right)$ : maximum reaction force expended to complete either left (or right) side-turn.

BACK Time (sec): time to return to one's back from either left (or right) side-turn.

$\mathrm{BL}(\mathrm{cm})$ : distance traveled upon returning to one's back after completing left (or right) side-turn.

BACK Speed $(\mathrm{cm} / \mathrm{sec})$ : speed (distance/time) of turning unto one's back from either left (or right) side-turn.

BACK Peak Time (ds): time to reach peak pressure (turning swiftness) when completing back-turn.

BACK Force $\left(\mathrm{kg} \cdot \mathrm{m} / \mathrm{s}^{2}\right)$ : maximum reaction force expended to complete the turn unto one's back from either left (or right) side-turn.

TURN Weight ratio: ratio of TURN force to weight.

BACK Weight ratio: ratio of BACK force to weight.

TABLE 3: Gender differences in turning ability.

\begin{tabular}{|c|c|c|c|c|c|}
\hline & \multicolumn{2}{|c|}{ Men } & \multicolumn{2}{|c|}{ Women } & \multirow[b]{2}{*}{$P$ value $^{\dagger}$} \\
\hline & $n^{\mathrm{a}}$ & Mean \pm SD & $n$ & Mean \pm SD & \\
\hline Turn time (sec) & 340 & $5.60 \pm 0.79$ & 240 & $5.91 \pm 1.29$ & 0.226 \\
\hline $\mathrm{TL}(\mathrm{cm})$ & 340 & $24.12 \pm 8.09$ & 240 & $24.81 \pm 9.22$ & 0.827 \\
\hline Turn speed $(\mathrm{cm} / \mathrm{sec})$ & 340 & $4.33 \pm 1.41$ & 240 & $4.36 \pm 1.93$ & 0.965 \\
\hline Turn peak time (ds) & 340 & $7.89 \pm 6.83$ & 240 & $8.77 \pm 7.96$ & 0.482 \\
\hline Turn force $\left(\mathrm{kg} \cdot \mathrm{m} / \mathrm{s}^{2}\right)$ & 340 & $70.80 \pm 11.89$ & 240 & $56.34 \pm 9.18$ & $0.002^{*}$ \\
\hline Back time (sec) & 340 & $5.23 \pm 1.98$ & 240 & $5.22 \pm 0.99$ & 0.947 \\
\hline $\mathrm{BL}(\mathrm{cm})$ & 340 & $24.35 \pm 8.14$ & 240 & $25.07 \pm 9.24$ & 0.823 \\
\hline Back speed $(\mathrm{cm} / \mathrm{sec})$ & 340 & $4.81 \pm 1.69$ & 240 & $4.95 \pm 2.02$ & 0.834 \\
\hline Back peak time (ds) & 340 & $11.59 \pm 13.09$ & 240 & $10.21 \pm 5.83$ & 0.331 \\
\hline Back force $\left(\mathrm{kg} \cdot \mathrm{m} / \mathrm{s}^{2}\right)$ & 340 & $71.42 \pm 12.74$ & 240 & $56.99 \pm 8.98$ & $0.002^{*}$ \\
\hline Turn-weight ratio & 340 & $1.015 \pm 0.108$ & 240 & $1.035 \pm 0.139$ & 0.642 \\
\hline Back-weight ratio & 340 & $1.023 \pm 0.121$ & 240 & $1.047 \pm 0.127$ & 0.595 \\
\hline
\end{tabular}

${ }^{a}$ Numbers of measurements; ${ }^{\dagger}$ determined by repeated measures ANOVA (within-subject effect).

${ }^{*}: P<0.05$ indicated statistical significance.

intraclass correlation coefficients (ICCs) [15]. An ICC $>0.75$ indicates excellent reliability [16]. The ICCs calculated were as follows: turn time, ICC $=0.609$; TL, ICC $=0.929$; turn speed, ICC $=0.866$; back time, ICC $=0.854 ;$ BL, ICC $=0.966$; back speed, ICC $=0.942$; turn peak time, ICC $=0.571$; back peak time, ICC $=0.835$; turn force, ICC $=0.993$; back force, ICC $=$ 0.998 ; turn-weight ratio, ICC $=0.983$; and back-weight ratio, ICC $=0.996$.
3.3. Comparison of Parameters Related to Turning Ability. There was no significant difference between left versus right side-turning (Table 2 ). Men had significantly higher turn force $(P=0.002)$ and back force $(P=0.002)$ when compared with women (Table 3 ). Healthy volunteers with BMI $\geq$ $27 \mathrm{~kg} / \mathrm{m}^{2}$ had significantly higher turn force $(P=0.007)$ and back force $(P=0.007)$ when compared with those who had BMI $<27 \mathrm{~kg} / \mathrm{m}^{2}$ (Table 4 ). Subjects $\geq 65$ years of age took 
TABLE 4: Association between BMI and turning ability of healthy volunteers.

\begin{tabular}{|c|c|c|c|c|c|}
\hline & \multicolumn{2}{|c|}{$\mathrm{BMI}<27 \mathrm{~kg} / \mathrm{m}^{2}$} & \multicolumn{2}{|c|}{$\mathrm{BMI} \geq 27 \mathrm{~kg} / \mathrm{m}^{2}$} & \multirow[b]{2}{*}{$P$ value $^{\dagger}$} \\
\hline & $n^{\mathrm{a}}$ & Mean \pm SD & $n$ & Mean \pm SD & \\
\hline Turn time (sec) & 440 & $5.71 \pm 1.08$ & 140 & $5.80 \pm 0.90$ & 0.782 \\
\hline $\mathrm{TL}(\mathrm{cm})$ & 440 & $23.30 \pm 7.85$ & 140 & $27.89 \pm 9.77$ & 0.203 \\
\hline Turn speed $(\mathrm{cm} / \mathrm{sec})$ & 440 & $4.16 \pm 1.57$ & 140 & $4.89 \pm 1.76$ & 0.292 \\
\hline Turn peak time (ds) & 440 & $8.16 \pm 7.06$ & 140 & $8.52 \pm 8.12$ & 0.802 \\
\hline Turn force $\left(\mathrm{kg} \cdot \mathrm{m} / \mathrm{s}^{2}\right)$ & 440 & $61.30 \pm 11.05$ & 140 & $75.86 \pm 12.40$ & $0.007^{*}$ \\
\hline Back time (sec) & 440 & $5.14 \pm 1.27$ & 140 & $5.51 \pm 2.47$ & 0.160 \\
\hline $\mathrm{BL}(\mathrm{cm})$ & 440 & $23.52 \pm 7.87$ & 140 & $28.19 \pm 9.82$ & 0.202 \\
\hline Back speed $(\mathrm{cm} / \mathrm{sec})$ & 440 & $4.70 \pm 1.71$ & 140 & $5.39 \pm 2.10$ & 0.366 \\
\hline Back peak time (ds) & 440 & $10.47 \pm 11.69$ & 140 & $12.75 \pm 6.57$ & 0.161 \\
\hline Back force $\left(\mathrm{kg} \cdot \mathrm{m} / \mathrm{s}^{2}\right)$ & 440 & $61.84 \pm 11.34$ & 140 & $76.80 \pm 12.96$ & $0.007^{*}$ \\
\hline Turn-weight ratio & 440 & $1.044 \pm 0.115$ & 140 & $0.957 \pm 0.123$ & 0.077 \\
\hline Back-weight ratio & 440 & $1.053 \pm 0.115$ & 140 & $0.969 \pm 0.131$ & 0.096 \\
\hline
\end{tabular}

${ }^{\mathrm{a}}$ Numbers of measurements; ${ }^{\dagger}$ determined by repeated measures ANOVA (within-subject effect).

${ }^{*}: P<0.05$ indicated statistical significance.

TABLE 5: Association between age and turning ability of healthy volunteers.

\begin{tabular}{|c|c|c|c|c|c|}
\hline & \multicolumn{2}{|c|}{ Age $<65$ year } & \multicolumn{2}{|c|}{ Age $\geq 65$ year } & \multirow{2}{*}{$P$ value $^{\dagger}$} \\
\hline & $n^{\mathrm{a}}$ & Mean \pm SD & $n$ & Mean \pm SD & \\
\hline Turn time (sec) & 460 & $5.65 \pm 1.03$ & 120 & $6.04 \pm 1.04$ & 0.221 \\
\hline $\mathrm{TL}(\mathrm{cm})$ & 460 & $25.26 \pm 8.80$ & 120 & $21.13 \pm 6.75$ & 0.280 \\
\hline Turn speed $(\mathrm{cm} / \mathrm{sec})$ & 460 & $4.57 \pm 1.72$ & 120 & $3.46 \pm 0.86$ & 0.127 \\
\hline Turn peak time (ds) & 460 & $7.57 \pm 6.88$ & 120 & $10.84 \pm 8.37$ & $0.025^{*}$ \\
\hline Turn force $\left(\mathrm{kg} \cdot \mathrm{m} / \mathrm{s}^{2}\right)$ & 460 & $66.17 \pm 12.73$ & 120 & $59.64 \pm 12.65$ & 0.276 \\
\hline Back time (sec) & 460 & $5.13 \pm 1.25$ & 120 & $5.60 \pm 2.64$ & 0.089 \\
\hline $\mathrm{BL}(\mathrm{cm})$ & 460 & $25.49 \pm 8.88$ & 120 & $21.40 \pm 6.57$ & 0.293 \\
\hline Back speed $(\mathrm{cm} / \mathrm{sec})$ & 460 & $5.09 \pm 1.88$ & 120 & $4.00 \pm 1.36$ & 0.167 \\
\hline Back peak time (ds) & 460 & $11.14 \pm 11.53$ & 120 & $10.56 \pm 6.78$ & 0.742 \\
\hline Back force $\left(\mathrm{kg} \cdot \mathrm{m} / \mathrm{s}^{2}\right)$ & 460 & $66.69 \pm 13.14$ & 120 & $60.71 \pm 13.25$ & 0.334 \\
\hline Turn-weight ratio & 460 & $1.040 \pm 0.108$ & 120 & $0.958 \pm 0.149$ & 0.114 \\
\hline Back-weight ratio & 460 & $1.048 \pm 0.109$ & 120 & $0.975 \pm 0.158$ & 0.173 \\
\hline
\end{tabular}

${ }^{a}$ Numbers of measurements; ${ }^{\dagger}$ determined by repeated measures ANOVA (within-subject effect).

${ }^{*}: P<0.05$ indicated statistical significance.

significantly longer time to reach peak swiftness (turn peak time) $(P=0.025)$ compared to those $<65$ years old (Table 5$)$.

3.4. Factors Associated with Parameters of Turning Ability: The GEE Model (Table 6). The GEE models included all variables in the multivariate analysis. After adjusting for other covariates, factors associated with parameters related to turning ability were as follows: (i) men had higher turn force $(P=$ $0.020)$ and back force $(P=0.041)$ than women; (ii) BMI positively correlated with higher TL $(P=0.047)$, turn force $(P<0.001)$, and back force $(P<0.001)$, respectively; (iii) age positively correlated with back time $(P=0.033)$ and negatively correlated with turn speed $(P=0.005)$, back speed $(P=$ $0.014)$, turn force $(P=0.010)$, and back force $(P=0.016)$, respectively; (iv) turning times negatively correlated with turn peak time (turning swiftness) $(P=0.008)$.

\section{Discussion}

Our mobility detection system was the first to quantify turning ability in the elderly subject, underscoring the originality of this research. In addition, we found that men exhibited significantly higher side-turning force and back-turning force compared with women. Healthy volunteers with $\mathrm{BMI} \geq 27 \mathrm{~kg} / \mathrm{m}^{2}$ had significantly higher side-turning force (and back-turning force) compared with those who had BMI $<27 \mathrm{~kg} / \mathrm{m}^{2}$. Interestingly, subjects $\geq 65$ years old took significantly longer to reach peak turning swiftness compared with those subjects $<65$ years old. Multivariate analysis showed that age positively correlated with back-turning time and negatively correlated with side-turning speed, back-turning speed, side-turning force, and back-turning force.

Slow turning speed in the elderly may arise from several causes including loss of skeletal muscle mass and force [17] as 
TABLE 6: Generalized predictors affecting turning ability.

\begin{tabular}{|c|c|c|c|c|}
\hline \multirow{2}{*}{ Variables } & \multicolumn{2}{|c|}{ Univariate } & \multicolumn{2}{|c|}{ Multivariate } \\
\hline & Beta $(95 \%$ CI $)$ & $P$ value & Beta $(95 \% \mathrm{CI})$ & $P$ value \\
\hline \multicolumn{5}{|l|}{ Turn time (sec) } \\
\hline Gender (men versus women) & $-0.310(-0.819,0.198)$ & 0.232 & $-0.414(-0.842,0.014)$ & 0.058 \\
\hline BMI (per kg/m²) & $0.004(-0.063,0.071)$ & 0.904 & $0.028(-0.022,0.078)$ & 0.267 \\
\hline Age (per year) & $0.010(-0.002,0.023)$ & 0.106 & $0.010(-0.002,0.022)$ & 0.114 \\
\hline Side-turn (right versus left) & $0.040(-0.255,0.334)$ & 0.792 & $0.040(-0.255,0.334)$ & 0.792 \\
\hline Times & $-0.007(-0.039,0.026)$ & 0.685 & $-0.007(-0.039,0.026)$ & 0.685 \\
\hline \multicolumn{5}{|l|}{$\mathrm{TL}(\mathrm{cm})$} \\
\hline Gender (men versus women) & $-0.697(-6.788,5.395)$ & 0.823 & $-2.990(-8.883,2.903)$ & 0.320 \\
\hline $\mathrm{BMI}($ per kg/m²) & $0.377(-0.323,1.076)$ & 0.291 & $0.673(0.010,1.336)$ & $0.047^{*}$ \\
\hline Age (per year) & $-0.125(-0.262,0.011)$ & 0.072 & $-0.137(-0.277,0.003)$ & 0.056 \\
\hline Side-turn (right versus left) & $0.116(-1.250,1.483)$ & 0.868 & $0.116(-1.250,1.483)$ & 0.868 \\
\hline Times & $0.089(-0.008,0.185)$ & 0.071 & $0.089(-0.008,0.185)$ & 0.071 \\
\hline \multicolumn{5}{|l|}{ Turn speed $(\mathrm{cm} / \mathrm{sec})$} \\
\hline Gender (men versus women) & $-0.027(-1.234,1.181)$ & 0.966 & $-0.377(-1.577,0.823)$ & 0.538 \\
\hline BMI $\left(\right.$ per $\left.1 \mathrm{~kg} / \mathrm{m}^{2}\right)$ & $0.058(-0.075,0.191)$ & 0.391 & $0.104(-0.017,0.226)$ & 0.093 \\
\hline Age (per 1 year) & $-0.031(-0.052,-0.009)$ & 0.005 & $-0.032(-0.055,-0.010)$ & $0.005^{*}$ \\
\hline Side-turn (right versus left) & $0.059(-0.130,0.248)$ & 0.539 & $0.059(-0.130,0.248)$ & 0.539 \\
\hline Times & $0.021(0.000,0.041)$ & 0.050 & $0.021(0.000,0.041)$ & 0.050 \\
\hline \multicolumn{5}{|l|}{ TURN Peak Time (ds) } \\
\hline Gender (men versus women) & $-0.878(-3.271,1.515)$ & 0.472 & $-1.235(-3.747,1.277)$ & 0.335 \\
\hline $\mathrm{BMI}\left(\right.$ per $\left.\mathrm{kg} / \mathrm{m}^{2}\right)$ & $0.036(-0.308,0.381)$ & 0.836 & $0.095(-0.267,0.457)$ & 0.606 \\
\hline Age (per year) & $0.053(-0.004,0.110)$ & 0.066 & $0.052(-0.003,0.107)$ & 0.066 \\
\hline Side-turn (right versus left) & $-0.382(-2.664,1.901)$ & 0.743 & $-0.382(-2.664,1.901)$ & 0.743 \\
\hline Times & $-0.185(-0.321,-0.049)$ & 0.008 & $-0.185(-0.321,-0.049)$ & $0.008^{*}$ \\
\hline \multicolumn{5}{|l|}{ TURN Force $\left(\mathrm{kg} \cdot \mathrm{m} / \mathrm{s}^{2}\right)$} \\
\hline Gender (men versus women) & $14.465(7.121,21.808)$ & 0.000 & $6.708(1.038,12.378)$ & $0.020^{*}$ \\
\hline BMI (per kg/m²) & $2.627(1.778,3.476)$ & $<0.0001$ & $2.237(1.432,3.042)$ & $<0.0001^{*}$ \\
\hline Age (per year) & $-0.117(-0.347,0.114)$ & 0.320 & $-0.160(-0.282,-0.039)$ & $0.010^{*}$ \\
\hline Side-turn (right versus left) & $0.651(-0.977,2.279)$ & 0.433 & $0.651(-0.977,2.279)$ & 0.433 \\
\hline Times & $-0.048(-0.108,0.012)$ & 0.119 & $-0.048(-0.108,0.012)$ & 0.119 \\
\hline \multicolumn{5}{|l|}{ BACK Time (sec) } \\
\hline Gender (men versus women) & $0.016(-0.411,0.442)$ & 0.943 & $-0.113(-0.442,0.217)$ & 0.503 \\
\hline BMI (per kg/m²) & $0.034(-0.030,0.098)$ & 0.303 & $0.035(-0.014,0.084)$ & 0.158 \\
\hline Age (per year) & $0.012(0.001,0.023)$ & 0.032 & $0.011(0.001,0.022)$ & $0.033^{*}$ \\
\hline Side-turn (right versus left) & $0.121(-0.247,0.488)$ & 0.520 & $0.121(-0.247,0.488)$ & 0.520 \\
\hline Times & $-0.006(-0.055,0.042)$ & 0.803 & $-0.006(-0.055,0.042)$ & 0.803 \\
\hline \multicolumn{5}{|l|}{$\mathrm{BL}(\mathrm{cm})$} \\
\hline Gender (men versus women) & $-0.722(-6.905,5.462)$ & 0.819 & $-3.025(-9.116,3.065)$ & 0.330 \\
\hline $\mathrm{BMI}($ per kg/m²) & $0.377(-0.336,1.089)$ & 0.300 & $0.676(-0.016,1.368)$ & 0.055 \\
\hline Age (per year) & $-0.127(-0.265,0.012)$ & 0.073 & $-0.138(-0.282,0.005)$ & 0.058 \\
\hline Side-turn (right versus left) & $-0.022(-1.320,1.276)$ & 0.973 & $-0.022(-1.320,1.276)$ & 0.973 \\
\hline Times & $0.039(-0.050,0.128)$ & 0.389 & $0.039(-0.050,0.128)$ & 0.389 \\
\hline \multicolumn{5}{|l|}{ BACK Speed $(\mathrm{cm} / \mathrm{sec})$} \\
\hline Gender (men versus women) & $-0.139(-1.426,1.148)$ & 0.833 & $-0.500(-1.758,0.757)$ & 0.436 \\
\hline $\mathrm{BMI}\left(\right.$ per $\mathrm{kg} / \mathrm{m}^{2}$ ) & $0.052(-0.096,0.199)$ & 0.492 & $0.108(-0.032,0.248)$ & 0.131 \\
\hline Age (per year) & $-0.032(-0.059,-0.006)$ & 0.016 & $-0.034(-0.061,-0.007)$ & $0.014^{*}$ \\
\hline
\end{tabular}


TABLE 6: Continued.

\begin{tabular}{|c|c|c|c|c|}
\hline \multirow{2}{*}{ Variables } & \multicolumn{2}{|c|}{ Univariate } & \multicolumn{2}{|c|}{ Multivariate } \\
\hline & Beta $(95 \% \mathrm{CI})$ & $P$ value & Beta $(95 \% \mathrm{CI})$ & $P$ value \\
\hline Turn side (right versus left) & $-0.053(-0.376,0.270)$ & 0.747 & $-0.053(-0.376,0.270)$ & 0.747 \\
\hline Times & $0.005(-0.016,0.026)$ & 0.631 & $0.005(-0.016,0.026)$ & 0.631 \\
\hline \multicolumn{5}{|l|}{ BACK Peak Time (ds) } \\
\hline Gender (men versus women) & $1.389(-1.105,3.882)$ & 0.275 & $0.473(-2.984,3.929)$ & 0.789 \\
\hline BMI $\left(\right.$ per $\left.\mathrm{kg} / \mathrm{m}^{2}\right)$ & $0.289(-0.047,0.625)$ & 0.092 & $0.264(-0.214,0.741)$ & 0.279 \\
\hline Age (per year) & $-0.010(-0.065,0.045)$ & 0.718 & $-0.015(-0.066,0.036)$ & 0.559 \\
\hline Side-turn (right versus left) & $-0.231(-2.757,2.296)$ & 0.858 & $-0.231(-2.757,2.296)$ & 0.858 \\
\hline Times & $-0.007(-0.331,0.316)$ & 0.965 & $-0.007(-0.331,0.316)$ & 0.965 \\
\hline \multicolumn{5}{|l|}{ BACK Force $\left(\mathrm{kg} \cdot \mathrm{m} / \mathrm{s}^{2}\right)$} \\
\hline Gender (men versus women) & $14.430(6.843,22.018)$ & 0.0002 & $6.380(0.262,12.497)$ & $0.041^{*}$ \\
\hline $\mathrm{BMI}($ per kg/m²) & $2.687(1.819,3.555)$ & $<0.0001$ & $2.321(1.467,3.175)$ & $<0.0001^{*}$ \\
\hline Age (per year) & $-0.116(-0.357,0.125)$ & 0.344 & $-0.161(-0.292,-0.030)$ & $0.016^{*}$ \\
\hline Side-turn (right versus left) & $0.847(-0.347,2.042)$ & 0.165 & $0.847(-0.347,2.042)$ & 0.165 \\
\hline Times & $0.032(-0.065,0.128)$ & 0.519 & $0.032(-0.065,0.128)$ & 0.519 \\
\hline \multicolumn{5}{|l|}{ TURN Weight ratio } \\
\hline Gender (men versus women) & $-0.020(-0.105,0.064)$ & 0.637 & $0.015(-0.065,0.096)$ & 0.708 \\
\hline BMI (per $\left.\mathrm{kg} / \mathrm{m}^{2}\right)$ & $-0.009(-0.021,0.002)$ & 0.112 & $-0.010(-0.022,0.001)$ & 0.082 \\
\hline Age (per year) & $-0.001(-0.003,0.001)$ & 0.440 & $-0.001(-0.003,0.001)$ & 0.540 \\
\hline Side-turn (right versus left) & $0.015(-0.014,0.044)$ & 0.302 & $0.015(-0.014,0.044)$ & 0.302 \\
\hline Times & $-0.001(-0.002,0.000)$ & 0.163 & $-0.001(-0.002,0.000)$ & 0.163 \\
\hline \multicolumn{5}{|l|}{ BACK Weight ratio } \\
\hline Gender (men versus women) & $-0.024(-0.108,0.061)$ & 0.5805 & $0.009(-0.077,0.094)$ & 0.844 \\
\hline BMI $\left(\right.$ per $\left.\mathrm{kg} / \mathrm{m}^{2}\right)$ & $-0.009(-0.021,0.003)$ & 0.1285 & $-0.009(-0.021,0.003)$ & 0.127 \\
\hline Age (per year) & $-0.001(-0.003,0.001)$ & 0.4668 & $-0.001(-0.003,0.002)$ & 0.550 \\
\hline Side-turn (right versus left) & $0.017(-0.004,0.037)$ & 0.1114 & $0.017(-0.004,0.037)$ & 0.111 \\
\hline Times & $0.000(-0.001,0.002)$ & 0.5404 & $0.000(-0.001,0.002)$ & 0.540 \\
\hline
\end{tabular}

well as loss of balance with increasing age [18]. Proprioceptive acuity also degrades over time in the older patient and has been directly correlated with falls and reduced functional independence [19]. It may also be a contributing factor in the reduction of turning speed with aging. Although such factors are understandable, this study is the first to present quantitative data on the decline in turning ability in the elderly. These novel findings can also be used to lay a foundation for monitoring those patients who have reduced turning ability due to long-term lack of activity, such as chronic bed-ridden patients, and can be further correlated with functional scales. In addition, they underscore the need for aggressive physical therapy, especially side-turning in the bed-ridden patient, as it has been shown that regular physical activity is an important strategy in attenuating age-related decay of muscle structure and function [17].

The ability to complete a side-turn is an important activity in bed-ridden patients [20], as a good side-turn can reduce the likelihood of developing bedsores [1], reverse deconditioning [21], and improve mobility [22]. Ability to complete a side-turn requires motor coordination involving balance, proprioception, and cerebellar function [23-28]. During infancy, this side-turning function is accomplished as an important first step in learning to walk without support; that is, the developmental stages include turning over, sitting, crawling, standing, and, finally, walking [26]. At approximately 4-6 months of age, the infant progresses from the ability to turn over to the ability to stand up, an action requiring coordination of nerve function, muscle strength, and bone support. Unfortunately, when this ability is impaired in bedridden patients such as stroke victims, or patients in the intensive care unit (ICU), it represents a decline in basic activity and functioning. However, no quantifiable clinical turning tool has yet been developed to monitor this decline in function.

The prevention of bedsores through side-turns is a very important and relevant topic. Several factors are involved in the development of bedsores and can be divided into intrinsic versus extrinsic factors. Intrinsic factors include nutrition and skin humidity, while extrinsic factors include direct skin pressure, shear forces, and/or friction [29]. Generally, direct pressure has the greatest impact on pressure sore development [4, $30,31]$. Therefore, it is clinically important to stand the patient up every two hours to prevent or treat pressure ulcers, but 
currently there is no particular requirement for side-turning that is quantifiable. Our system allows for such monitoring as well as monitoring the frequency of side-turns.

Once bedsores develop, they represent an acute health condition that results in increased cost and suffering over months or even years. Effective ulcer prevention and early detection is, therefore, necessary. Strong motivation for a computerized mobility detection system arises from the growing shortage of trained health care providers [32] and the ever-increasing cost of health care. One particular multimodal approach to monitoring sleeping posture, using a pressure sensor array and video camera as complementary modalities, has been proposed [7,33]. Unfortunately, the use of cameras in nursing units entails certain privacy issues which must be considered and camera surveillance also involves nursing time which tends to drain an already encumbered health care system [7, 32].

4.1. Study Limitations. Our study had several limitations. Participants were all healthy volunteers with a mean age of $46.1 \pm$ $19.64 \mathrm{yrs}$ (ranging from 23 to $86 \mathrm{yrs}$ ). Thus, no patients at risk for developing bed sores (such as stroke, spinal cord injury, or ICU patients) were included in the study. Further validation of our method in patients with (or at high risk for) impaired side-turning on beds is required. Overnight monitoring of older healthy individuals and patients using our mobility detection system is also required for complete assessment. Reliability of the mobility detection system should also be examined by video monitoring system. Since this is the first study of its kind to analyze turning swiftness, further clinical trials examining a larger aging population are required to confirm our results. In particular, turn angle, turn angle speed, and other related measures should be considered in future clinical trials since they are less dependent on the height or width of the participants.

\section{Conclusion}

Our mobility detection system has proven to be effective in detecting changes in turning swiftness on beds. It is effective and easy to use. However, further validation with patients at high risk for impaired side-turning on beds is required.

\section{Conflict of Interests}

The authors declare that there is no conflict of interests regarding the publication of this paper.

\section{Authors' Contribution}

Wen-Hsu Sung and Shun-Hwa Wei contributed equally to this study.

\section{Acknowledgments}

This study was supported by the National science council (no. NSC 101-2221-E-016-001) and Tri-Service General Hospital (No. TSGH-C101-110). The funding source had no role in study design, data collection and analysis, decision to publish, or preparation of the paper.

\section{References}

[1] M. Reddy, S. S. Gill, and P. A. Rochon, "Preventing pressure ulcers: a systematic review," Journal of the American Medical Association, vol. 296, no. 8, pp. 974-984, 2006.

[2] N. Graves, F. Birrell, and M. Whitby, "Effect of pressure ulcers on length of hospital stay," Infection Control and Hospital Epidemiology, vol. 26, no. 3, pp. 293-297, 2005.

[3] D. R. Lowne and M. Tarler, "Designing a low-cost mattress sensor for automated body position classification," in Proceedings of the 27th Annual International Conference of the Engineering in Medicine and Biology Society (IEEE-EMBS '05), pp. 6437-6440, New York, NY, USA, September 2005.

[4] J. A. Russell and S. L. Lichtenstein, "Randomized controlled trial to determine the safety and efficacy of a multi-cell pulsating dynamic mattress system in the prevention of pressure ulcers in patients undergoing cardiovascular surgery," Ostomy Wound Management, vol. 46, no. 2, pp. 46-55, 2000.

[5] C. C. Hsia, K. J. Liou, A. P. W. Aung, V. Foo, W. Huang, and J. Biswas, "Analysis and comparison of sleeping posture classification methods using pressure sensitive bed system," in Proceedings of the 31st Annual International Conference of the IEEE Engineering in Medicine and Biology Society (EMBC '09), pp. 6131-6134, September 2009.

[6] S. M. Motamedi, J. de Grood, S. Harman et al., "The effect of continuous pressure monitoring on strategic shifting of medical inpatients at risk for PUs," Journal of Wound Care, vol. 21, pp. 517-518, 2012.

[7] R. Yousefi, S. Ostadabbas, M. Faezipour et al., "Bed posture classification for pressure ulcer prevention," in Proceedings of the 33rd Annual International Conference of the IEEE Engineering in Medicine and Biology Society (EMBS '11), pp. 7175-7178, September 2011.

[8] C. J. Brown, D. L. Roth, and R. M. Allman, "Validation of use of wireless monitors to measure levels of mobility during hospitalization," Journal of Rehabilitation Research and Development, vol. 45, no. 4, pp. 551-558, 2008.

[9] B. M. Bates-Jensen, M. Cadogan, J. Jorge, and J. F. Schnelle, "Standardized quality-assessment system to evaluate pressure ulcer care in the nursing home," Journal of the American Geriatrics Society, vol. 51, no. 9, pp. 1195-1202, 2003.

[10] C.-C. Yang and Y.-L. Hsu, "A review of accelerometry-based wearable motion detectors for physical activity monitoring," Sensors, vol. 10, no. 8, pp. 7772-7788, 2010.

[11] Z. Zhang and S. Poslad, "Design and test of a hybrid foot force sensing and GPS system for richer user mobility activity recognition," Sensors, vol. 13, pp. 14918-14953, 2013.

[12] D. C. Ranasinghe, R. L. Shinmoto Torres, K. Hill, and R. Visvanathan, "Low cost and batteryless sensor-enabled radio frequency identification tag based approaches to identify patient bed entry and exit posture transitions," Gait \& Posture, vol. 39, pp. 118-123, 2014.

[13] F. Bagalà, J. Klenk, A. Cappello, L. Chiari, C. Becker, and U. Lindemann, "Quantitative description of the lie-to-sit-tostand-to-walk transfer by a single body-fixed sensor," IEEE Transactions on Neural Systems and Rehabilitation Engineering, vol. 21, pp. 624-633, 2013. 
[14] H. Wind, V. Gouttebarge, P. P. F. M. Kuijer, and M. H. W. FringsDresen, "Assessment of functional capacity of the musculoskeletal system in the context of work, daily living, and sport: a systematic review," Journal of Occupational Rehabilitation, vol. 15 , no. 2, pp. 253-272, 2005.

[15] P. E. Shrout and J. L. Fleiss, "Intraclass correlations: uses in assessing rater reliability," Psychological Bulletin, vol. 86, no. 2, pp. 420-428, 1979.

[16] J. L. Fleiss, Statistical Methods for Rates and Proportions, Wiley \& Sons, New York, NY, USA, 1981.

[17] S. Zampieri, L. Pietrangelo, S. Loefler et al., "Lifelong physical exercise delays age-associated skeletal muscle decline," The Journals of Gerontology A, 2014.

[18] N. Kanekar and A. S. Aruin, "Aging and balance control in response to external perturbations: role of anticipatory and compensatory postural mechanisms," Age, 2014.

[19] K. J. Suetterlin and A. A. Sayer, "Proprioception: where are we now? A commentary on clinical assessment, changes across the life course, functional implications and future interventions," Age and Ageing, 2013.

[20] C. Winkelman, P. A. Higgins, and Y.-J. K. Chen, "Activity in the chronically critically ill," Dimensions of Critical Care Nursing, vol. 24, no. 6, pp. 281-290, 2005.

[21] T. E. Rudy, J. R. Boston, S. J. Lieber, J. A. Kubinski, and A. Delitto, "Body motion patterns during a novel repetitive wheel-rotation task: a comparative study of healthy subjects and patients with low back pain," Spine, vol. 20, no. 23, pp. 2547-2554, 1995.

[22] N. Cullum and E. Petherick, "Pressure ulcers," Clinical Evidence, 2008.

[23] D. J. Serrien, Y. Li, M. S. F. Debaere, and S. P. Swinnen, "Proprioceptive regulation of interlimb behavior: interference between passive movement and active coordination dynamics," Experimental Brain Research, vol. 140, no. 4, pp. 411-419, 2001.

[24] W. T. Thach, "A role for the cerebellum in learning movement coordination," Neurobiology of Learning and Memory, vol. 70, no. 1-2, pp. 177-188, 1998.

[25] L. Mouchnino, R. Aurenty, J. Massion, and A. Pedotti, "Coordination between equilibrium and head-trunk orientation during leg movement: a new strategy built up by training," Journal of Neurophysiology, vol. 67, no. 6, pp. 1587-1598, 1992.

[26] M. de Onis, "Assessment of sex differences and heterogeneity in motor milestone attainment among populations in the WHO Multicentre Growth Reference Study," Acta Paediatrica, vol. 95, no. 450 , pp. 66-75, 2006.

[27] H. Sveistrup, S. Schneiberg, P. A. McKinley, B. J. McFadyen, and M. F. Levin, "Head, arm and trunk coordination during reaching in children," Experimental Brain Research, vol. 188, no. 2, pp. 237-247, 2008.

[28] E. M. Horn, S. F. Warren, and H. A. Jones, "An experimental analysis of a neurobehavioral motor intervention," Developmental Medicine and Child Neurology, vol. 37, no. 8, pp. 697-714, 1995.

[29] National Pressure Ulcer Advisory Panel, http://www.npuap .org/pr2.htm.

[30] M. Kosiak, "Etiology of decubitus ulcers," Archives of physical medicine and rehabilitation, vol. 42, pp. 19-29, 1961.

[31] J. B. Reswick and J. Rogers, "Experience at Ranchos Los Amigos Hospital with devices and techniques to prevent pressure sores," in Bedsore Biomechanics, R. M. Kenedi, J. M. Cowden, and J. T. Scales, Eds., pp. 301-310, Macmillan Press, London, UK, 1976.
[32] US Department of Health and Human Services, "Projected Supply Demand and Shortage of Registered Nurses: 20002020," 2002, http://www.ahcancal.org/research_data/staffing/ Documents/Registered_Nurse_Supply_Demand.pdf.

[33] W. Huang, A. Phyowai, S. Fook Foo et al., in Proceedings of the $I C P R$, vol. Multimodal sleeping posture classification, pp. 43364339, 2010. 


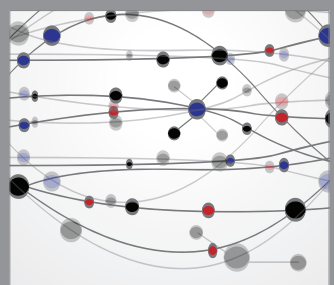

The Scientific World Journal
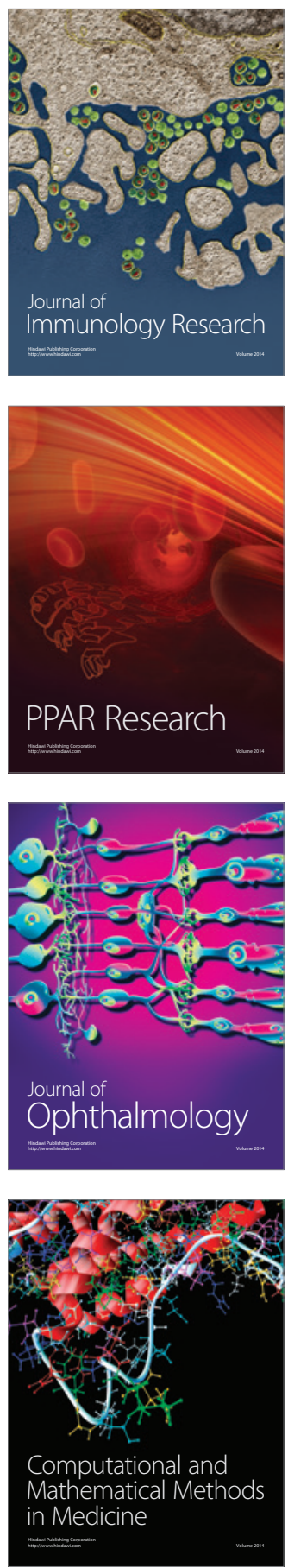

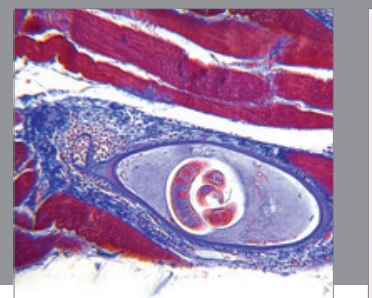

Gastroenterology

Research and Practice
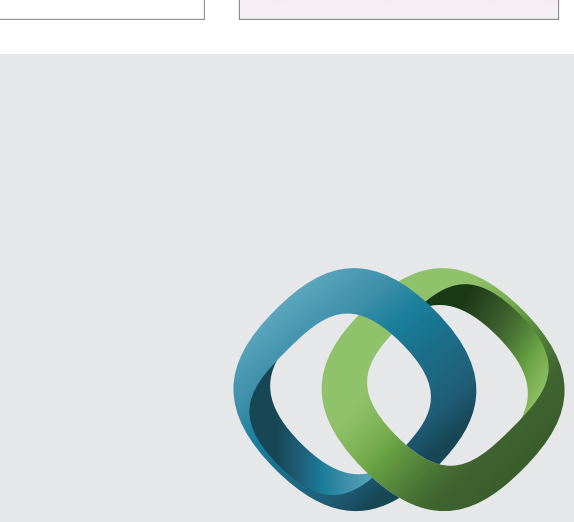

\section{Hindawi}

Submit your manuscripts at

http://www.hindawi.com
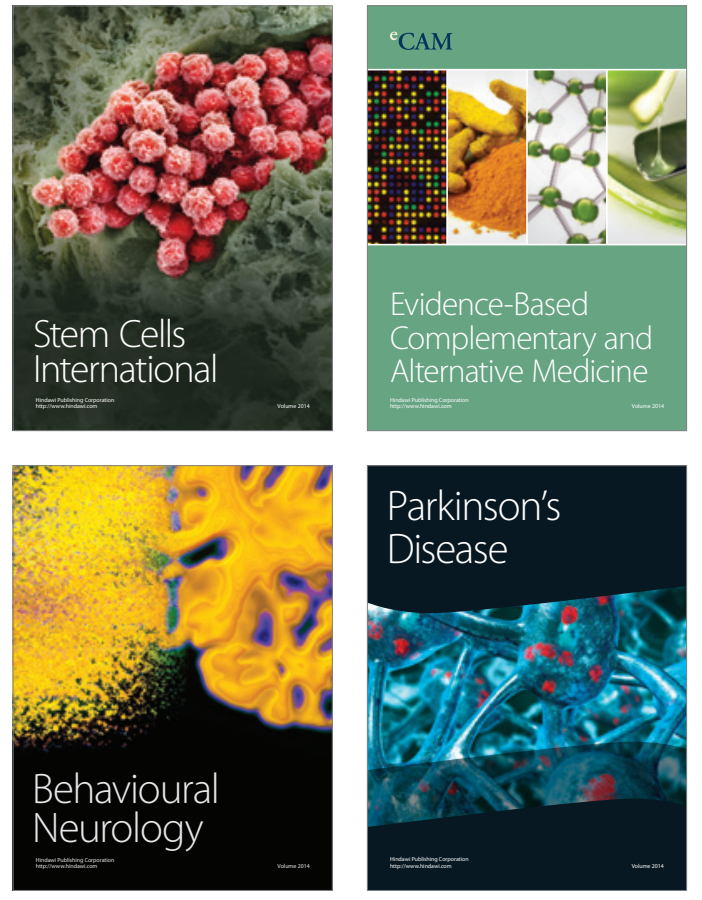
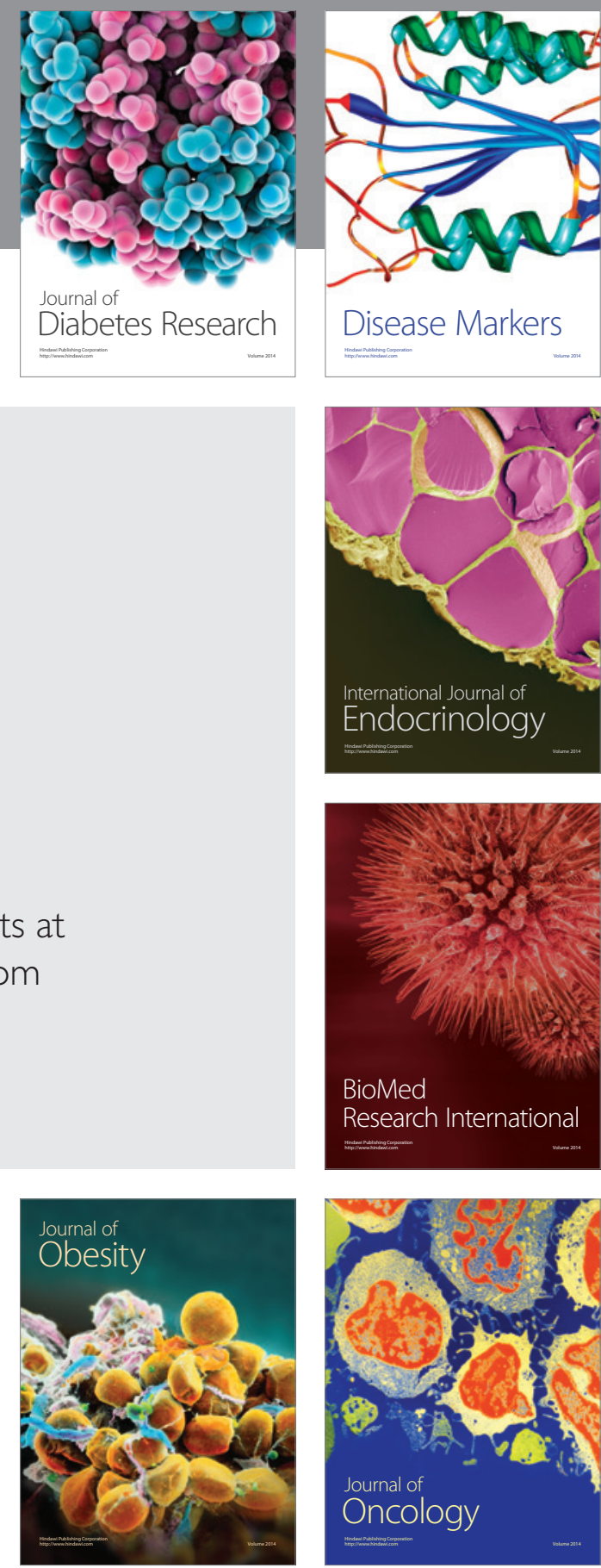

Disease Markers
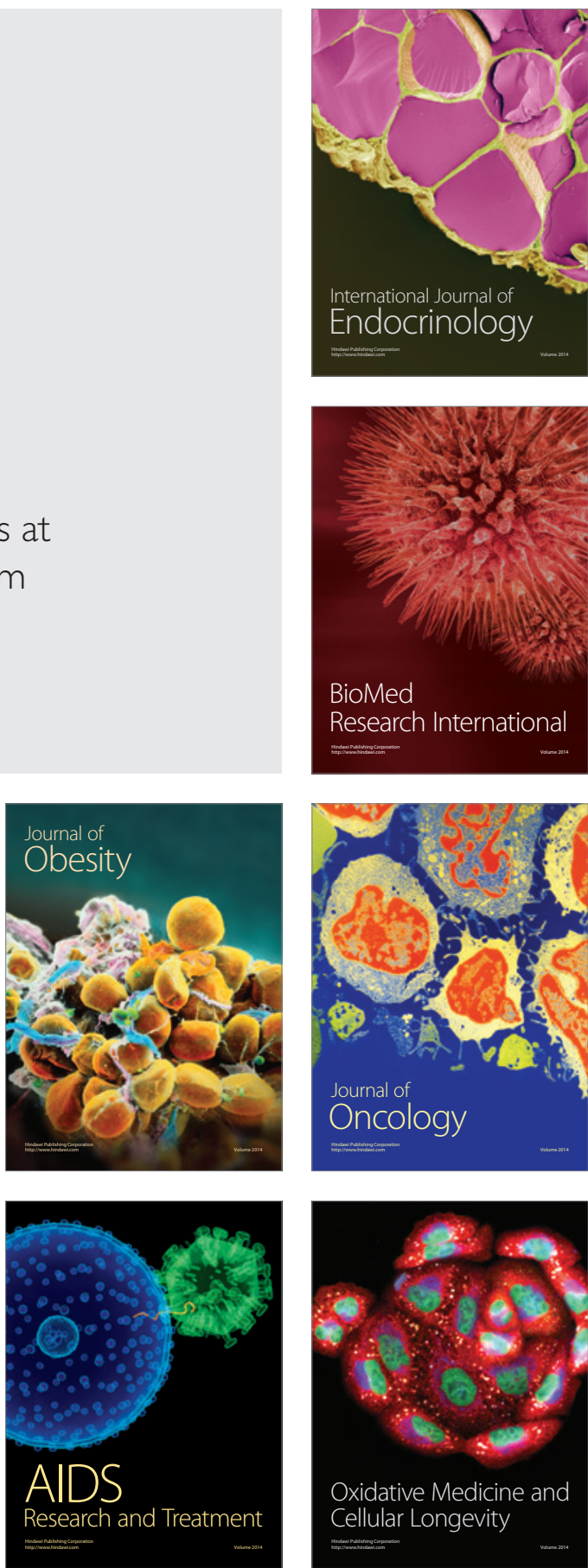